\title{
Colorectal carcinomas with KRAS mutation are associated with distinctive morphological and molecular features
}

Christophe Rosty ${ }^{1,2,3}$, Joanne P Young ${ }^{1,2}$, Michael D Walsh ${ }^{1,2}$, Mark Clendenning ${ }^{1}$, Rhiannon J Walters ${ }^{1}$, Sally Pearson ${ }^{1}$, Erika Pavluk ${ }^{1}$, Belinda Nagler ${ }^{1}$, David Pakenas ${ }^{1}$, Jeremy R Jass ${ }^{4,4}$, Mark A Jenkins ${ }^{5}$, Aung Ko Win ${ }^{5}$, Melissa C Southey ${ }^{6}$, Susan Parry ${ }^{7}$, John L Hopper ${ }^{5}$, Graham G Giles ${ }^{8}$, Elizabeth Williamson ${ }^{5}$, Dallas R English ${ }^{5,8}$ and Daniel D Buchanan ${ }^{1}$

${ }^{1}$ Cancer and Population Studies Group, Queensland Institute of Medical Research, Herston, Queensland, Australia; ${ }^{2}$ University of Queensland, School of Medicine, Herston, Queensland, Australia; ${ }^{3}$ Envoi Pathology, Herston, Queensland, Australia; ${ }^{4}$ Department of Cellular Pathology, St Marks Hospital, Harrow, UK; ${ }^{5}$ Centre for Molecular, Environmental, Genetic and Analytic Epidemiology, School of Population Health, The University of Melbourne, Carlton, Victoria, Australia; ${ }^{6}$ Genetic Epidemiology Laboratory, Department of Pathology, The University of Melbourne, Carlton, Victoria, Australia; ${ }^{7}$ Department of Gastroenterology, Middlemore Hospital, Auckland, New Zealand and ${ }^{8}$ Cancer Epidemiology Centre, Cancer Council Victoria, Carlton, Victoria, Australia

KRAS-mutated carcinomas comprise $35-40 \%$ of all colorectal carcinomas but little is known about their characteristics. The aim of this study was to examine the pathological and molecular features of KRAS-mutated colorectal carcinomas and to compare them with other carcinoma subgroups. KRAS mutation testing was performed in 776 incident tumors from the Melbourne Collaborative Cohort Study. $O^{6}$-methylguanine DNA methyltransferase (MGMT) status was assessed using both immunohistochemistry and MethyLight techniques. Microsatellite instability (MSI) phenotype and BRAF V600E mutation status were derived from earlier studies. Mutation in KRAS codon 12 or codon 13 was present in $28 \%$ of colorectal carcinomas. Compared with KRAS wildtype carcinomas, KRAS-mutated carcinomas were more frequently observed in contiguity with a residual polyp (38 vs 21\%; $P<0.001$ ), demonstrated mucinous differentiation (46 vs $31 \% ; P=0.001$ ) and were associated with different MSI status $(P<0.001)$ and with MGMT methylation (47 vs $21 \% ; P=0.001)$. Compared with tumors demonstrating neither BRAF nor KRAS mutation, KRAS-mutated carcinomas showed more frequent location in the proximal colon (41 vs 27\%; $P=0.001$ ), mucinous differentiation (46 vs $25 \%$; $P<0.001$ ), presence of a contiguous polyp (38 vs $22 \%$; $P<0.001$ ), MGMT methylation (47 vs 26\%; $P=0.01$ ) and loss of MGMT immunohistochemical expression (27 vs $19 \% ; P=0.02)$. KRAS-mutated carcinomas were distributed in a bimodal pattern along the proximal-distal axis of the colorectum. Compared with male subjects, female subjects were more likely to have KRAS-mutated carcinoma in the transverse colon and descending colon (39 vs $15 \% ; P=0.02$ ). No difference in overall survival was observed in patients according to their tumor KRAS mutation status. In summary, KRAS-mutated carcinomas frequently develop in contiguity with a residual polyp and show molecular features distinct from other colorectal carcinomas, in particular from tumors with neither BRAF nor KRAS mutation.

Modern Pathology (2013) 26, 825-834; doi:10.1038/modpathol.2012.240; published online 25 January 2013

Keywords: BRAF mutation; colorectal cancer; colorectal polyp; KRAS mutation; MGMT; molecular pathology; survival analysis

Correspondence: Dr C Rosty, MD, PhD, FRCPA, Cancer and Population Studies Group, Bancroft Centre, Queensland Institute of Medical Research, Herston, Queensland 4006, Australia.

E-mail: c.rosty@uq.edu.au.

Deceased.

Received 26 July 2012; revised 28 December 2012; accepted 28

December 2012; published online 25 January 2013
During the past two decades, colorectal carcinoma has evolved from being viewed as a single entity to a heterogeneous group of diseases classified into molecular groups with clinical utility and epidemiological relevance. ${ }^{1,2}$ Examples of this are seen in the recognition of Lynch syndrome using specific pathological features, ${ }^{3}$ and the roles of lifestyle and 
environmental factors in the development of colorectal carcinoma subsets. ${ }^{4,5}$ The complex interaction between an individual's genetic background and the environment has led to the concept of tumor uniqueness and molecular pathological epidemiology. ${ }^{6}$

Current colorectal carcinoma classification largely reflects the polyp of origin for each group, various molecular pathways to neoplastic progression and molecular correlates with epidemiological features. Among the most commonly altered molecular pathways in colorectal carcinoma, the RAS-MAPK signaling pathway is deregulated in $55 \%$ of tumors, comprising mutually exclusive mutations in $B R A F$, KRAS and NRAS. ${ }^{7}$ Colorectal carcinomas with $B R A F$ mutation have emerged as a distinct group with a combination of features that sets them apart from the remainder of colorectal carcinoma, including predilection for the proximal colon, female gender, high histological grade, high levels of $\mathrm{CpG}$ island methylator phenotype (CIMP-high), an origin in serrated polyps and association with increased body size.1,8,9 Somatic-activating mutation in the KRAS proto-oncogene is observed in $35-40 \%$ of colorectal carcinoma. ${ }^{7,10,11}$ The overwhelming majority of KRAS mutations (90\%) occur in codons 12 and 13 of exon 2, with the most frequent alteration being a $\mathrm{G}>\mathrm{A}$ transition in codon $12 .{ }^{12}$ The clinical significance of KRAS mutation in colorectal carcinoma patients is controversial; some studies reported no association with survival, ${ }^{13,14}$ whereas others suggested that patients with KRASmutated colorectal carcinoma have poorer outcome for any mutation subtype, ${ }^{15}$ mutation in codon 12 only ${ }^{16,17}$ or codon 13 only. ${ }^{11}$ There is more definitive evidence for a negative predictive value of KRAS mutation in patients with advanced stage disease treated with targeted anti-EGFR monoclonal antibody drugs. ${ }^{18}$

In the traditional adenoma-carcinoma pathway of colorectal carcinoma, KRAS mutation occurs early in the sequential neoplastic progression, after deregulation of the WNT pathway, commonly by $A P C$ mutation and before TP53 inactivation. ${ }^{19}$ However, KRAS mutation is not universally found in tumors harboring $A P C$ and TP53 mutations. ${ }^{20} \mathrm{In}$ addition, some studies have reported that KRASmutated colorectal carcinomas are associated with other distinctive molecular findings, including inactivation of the DNA repair gene $O^{6}$-methylguanine DNA methyltransferase (MGMT), and the poorly defined categories of low/indeterminate level of MSI and CIMP. ${ }^{21,22}$ Taken together, these observations suggest that some KRAS-mutated colorectal carcinomas may develop through a divergent molecular sequence.

Given that new associations have been uncovered when colorectal carcinomas were stratified for the presence of BRAF mutation, the possibility exists that KRAS-mutated tumors may also show currently unrecognized features and risk factor associations, which set them apart from the balance of colorectal carcinomas. In this report, we provide some important insights into the characteristics and origins of KRAS-mutated carcinoma and their gender-related distribution in the large bowel, which may contribute to a better understanding of the molecular epidemiology of colorectal carcinoma.

\section{Patients and methods}

\section{Subjects}

Participants were enrolled in The Melbourne Collaborative Cohort Study, which is a prospective study of 41514 people (17045 male subjects and 24469 female subjects) recruited between 1990 and 1994, with the aim of examining the role of lifestyle factors in the risk of cancer and heart disease. Participants were between 27 and 75 years of age (almost all were between the ages 40 and 69 years) at baseline. ${ }^{23}$ Incident cases of carcinoma of the colon or the rectum were identified by linkage to the populationbased cancer registries in Victoria and other Australian states. The study protocol was approved by the Cancer Council Victoria's Human Research Ethics Committee and the Human Research Ethics Committee of the Queensland Institute of Medical Research under protocol P799. Participants gave written consent for participation and for the investigators to obtain their medical records. Clinical data were collected from medical charts, colonoscopy and pathology reports. Location in the colon was designated as proximal colon for tumors located in the cecum, ascending colon and transverse colon, and as distal colon for tumors in the descending colon and sigmoid colon.

\section{Molecular Pathology}

All cases underwent standardized histopathology review by a specialist gastrointestinal pathologist (JRJ or CR). When patients received neoadjuvant therapy for a rectal carcinoma, the original biopsy sample was used for analysis. If the biopsy was not available, the case was excluded from the study. Tumors were assessed histologically for the following features: histological type, proportion of tumor with mucinous differentiation, grade, margin (circumscribed or infiltrative), tumor budding (non-quantitative method), the presence of tumorinfiltrating lymphocytes, and the presence and the histological type of a residual polyp adjacent to the carcinoma. Mucinous differentiation in the tumor was defined by the presence of pools of extracellular mucin-containing clusters of carcinomatous cells or individual tumor cells including signet ring cells. When $>50 \%$ of analyzed tumor demonstrated mucinous differentiation, the tumor was classified as mucinous carcinoma. Histological grading was performed using the latest WHO criteria. ${ }^{24}$ For conventional adenocarcinoma ('adenocarcinoma, 
NOS'), tumors were classified as low grade if $\geq 50 \%$ gland formation was present and high grade if $<50 \%$ gland formation was present. For mucinous carcinoma, tumors were graded according to the MSI phenotype: low grade if high level of MSI was present, high grade if no MSI-high was present. Residual polyps with a villous component comprised tubulovillous adenoma, villous adenoma and traditional serrated adenoma.

Somatic mutations in codons 12 and 13 of KRAS were screened using real-time PCR with high-resolution melting analysis in the presence of the SYTO9 fluorescent intercalating dye followed by direct Sanger sequencing on cases with differential melting profiles as described previously. ${ }^{25} B R A F$ V600E mutation analysis was performed using a real-time PCR-based allelic discrimination method. ${ }^{4}$ MSI status was determined using a 10-loci panel in tumor DNA and matched normal tissue DNA (BAT25, BAT26, BAT40, MYCL, D5S346, D17S250, ACTC, D18S55, D10S197 and BAT34C4) as described previously. ${ }^{26}$ Tumors were classified as microsatellite stable ( $0 \%$ of unstable loci), MSI-low/indeterminate ( $>0$ to $<30 \%$ of unstable loci) or MSI-high ( $\geq 30 \%$ of unstable loci). MGMT methylation was assessed using techniques described elsewhere. ${ }^{27}$

\section{Immunohistochemical Testing}

Immunohistochemistry for MGMT (clone MT3.1, 1:100 dilution; Fisher Scientific, Pittsburgh, PA, USA) was performed on formalin-fixed paraffinembedded tissue using an automated platform (Dako Autostainer; Dako, Carpinteria, CA, USA). Sections $(4 \mu \mathrm{m})$ were routinely dewaxed and rehydrated, and then subjected to heat-induced epitope retrieval in High pH Target Retrieval solution (Dako) using a pressure cooker, followed by MACH3 mouse HRP detection system (Biocare Medical, Concord, CA, USA). Antigenic sites were developed using DAB + liquid chromogen, and then the sections were counterstained with hematoxylin before mounting. Histologically normal colonic mucosa served as positive control tissue for MGMT expression. Stained sections were scored by two observers (CR and MDW) blinded to clinical and molecular testing results for cases. MGMT immunohistochemistry was scored as follows: (1) complete loss of expression; (2) reduced expression in tumor cells in comparison with normal epithelial and stromal cells; (3) tumor and non-tumor cells showed equivalent staining intensity; and (4) tumor cells showed increased staining intensity in comparison with non-tumor cells. For the analysis, only tumors showing a score 1 were interpreted as having abnormal loss of MGMT expression.

\section{Statistical Analysis}

Statistical analyses were performed with SPSS statistics software version 19.0 (SPSS, Chicago, IL,
USA) and Stata version 11.1 (StataCorp LP, College Station, TX, USA). Age is summarized by mean \pm s.d. Comparisons for categorical variables were performed using Pearson's $\chi^{2}$ test or Fisher's exact test where appropriate. Student's $t$-test was used to compare continuous variables between groups. Risk factor associations were initially examined uncorrected for multiple testing. They were then re-examined using a Bonferroni correction to guard against false-positive results.

Kaplan-Meier methods were used to estimate the survival curves by KRAS status (wild-type vs mutated), with death from all causes as the end point and time measured from the date of surgery until either death or the end of 2008. Survival curves were compared using the log-rank test. Cox proportional hazards multivariable regression models were used to estimate the hazard ratios (HR) associated with KRAS (wild-type vs mutated), adjusting for: sex, age at diagnosis $(<60,60-70,>70)$, tumor location (proximal vs distal), histological grade (high vs low), MSI status (MSI-high vs non MSIhigh), MGMT expression (normal vs absent) and $B R A F$ (wild-type vs mutated). This Cox model was refitted splitting the KRAS mutations into codons 12 and 13. We then excluded patients with a $B R A F$ mutation and refitted both these Cox models. Results are presented as estimated HRs with 95\% confidence intervals (CIs) and Wald test $P$-values. The proportional hazards assumption was assessed using graphical methods and tests based on Schoenfeld residuals. A two-tailed $P$-value was used for all analyses and values $<0.05$ were considered to be statistically significant.

\section{Results}

By the end of 2009 after excluding 182 subjects with colorectal carcinoma diagnoses pre-baseline, 1098 participants had at least one incident tumor. For subjects with multiple incident tumors, one was randomly selected for analysis. Data were available for 776 subjects $(71 \%)$. Reasons for subject exclusion from this study were unavailability of tissue sample $(n=228)$, insufficient DNA sample $(n=50)$ and unsatisfactory results $(n=44)$.

KRAS mutation was observed in $28 \%$ of colorectal carcinomas (87\% in codon 12 and $13 \%$ in codon 13; Table 1 and Supplementary Table). The most common variant in codon 12 was the c. $35 \mathrm{G}>\mathrm{A}$ transition (99/190, 52\%), followed by the c.35 G > T transversion $(58 / 190,31 \%)$. There were four tumors demonstrating mutations in both KRAS and BRAF; these cases were excluded from the analysis. KRASmutated carcinomas were compared with nonKRAS-mutated carcinomas for age, sex, histological features and molecular characteristics (Table 1). In addition, given that non-KRAS-mutated tumors included a distinct subset characterized by $B R A F$ mutation, analyses were also performed to compare 
Table 1 Summary of clinical, pathological and molecular features for 776 colorectal carcinomas from the Melbourne Collaborative Cohort Study

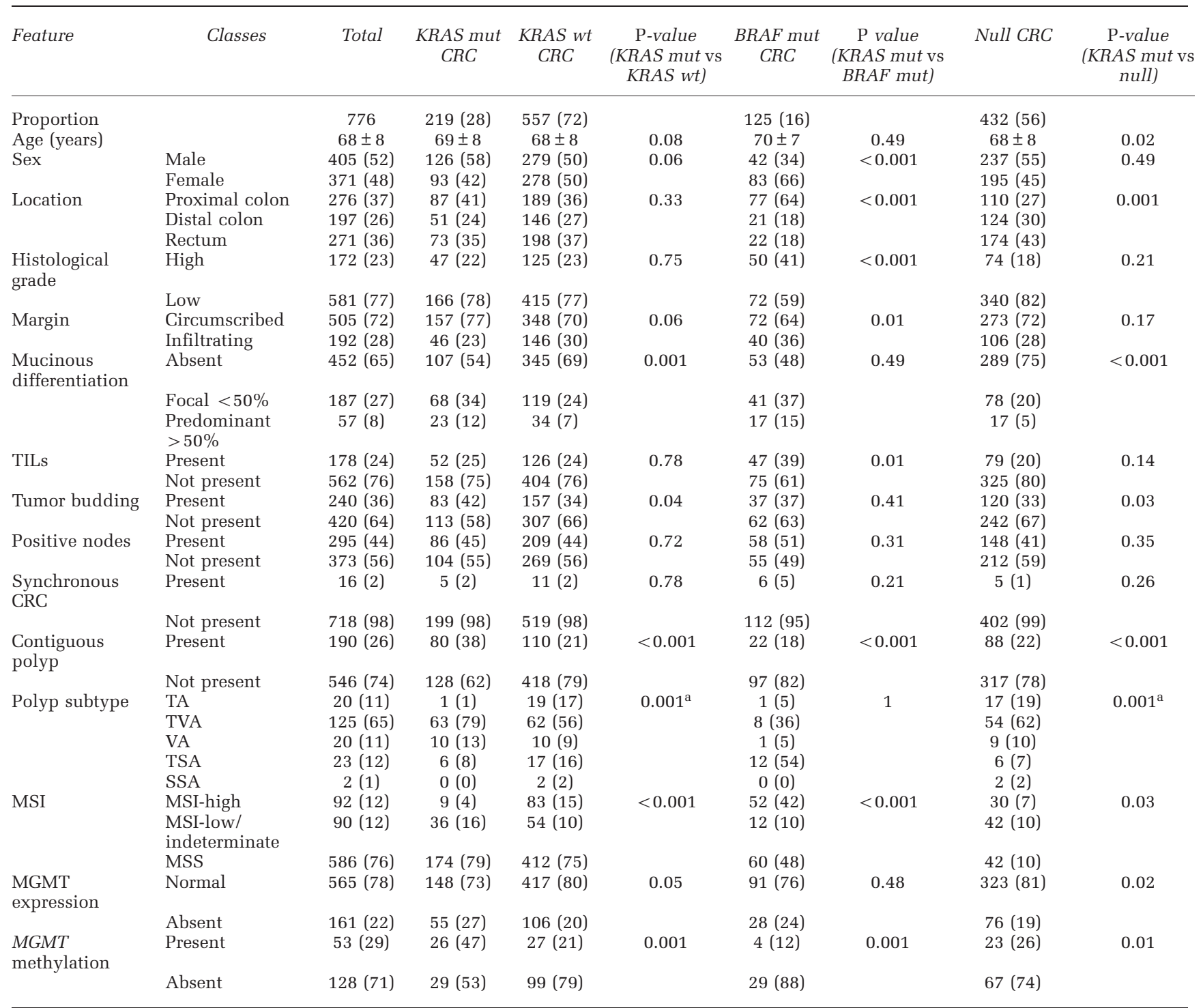

CRC: colorectal carcinoma; mut: mutation; wt: wild type; null: neither KRAS nor BRAF mutation; TIL: tumor-infiltrating lymphocytes; TA: tubular adenoma; TVA: tubulovillous adenoma; VA: villous adenoma; TSA: traditional serrated adenoma; SSA: sessile serrated adenoma; MSI: Microsatellite instability; MSS: Microsatellite stable.

KRAS-mutated tumors were initially compared with all other tumors. Given that KRAS wild-type carcinomas include a distinct subset of tumors characterized by BRAF mutation, analyses were also performed to compare $K R A S$-mutated carcinomas with both $B R A F$-mutated carcinomas and the remaining subset of tumors, which demonstrated neither somatic oncogene mutation (null carcinomas).

Results are given in absolute number and (percentage), unless otherwise specified. Age results are given in mean \pm s.d.

The $P$-value for significance was adjusted using the Bonferroni correction for multiple hypothesis testing of 17 variables (16 comparisons in Table 1 and one comparison in Table 2) to $P=0.05 / 34=0.0015$.

${ }^{a} P$-value comparing contiguous polyps with a villous architecture (TVA, VA and TSA) to the other polyp subtypes.

KRAS-mutated tumors with both $B R A F$-mutated tumors and the remaining subset of colorectal carcinomas, which demonstrated neither somatic oncogene mutation (hereafter referred to as 'null carcinomas'). The mean age at presentation for $K R A S$-mutated carcinoma was $69 \pm 8$ years, which was no significantly different to that for $B R A F$ mutated carcinoma at $70 \pm 7$ years and null carcinoma at $68 \pm 8$ years.

\section{Colon Site and Gender Distribution}

Gender distribution did not differ significantly between KRAS-mutated colorectal carcinomas and null carcinomas. However, women were over-represented in the $B R A F$-mutated carcinoma subset when compared with both KRAS-mutated carcinomas and null carcinomas $(66 \%$ vS $42 \%$ and $45 \%$, respectively; $P<0.001$ for $K R A S$ - vs $B R A F$-mutated 
Table 2 Summary of distribution of KRAS-mutated carcinomas, $B R A F$-mutated carcinomas and null carcinomas (neither KRAS nor BRAF mutation) by colonic site for female subjects, male subjects and both genders

\begin{tabular}{|c|c|c|c|c|c|}
\hline $\begin{array}{l}\text { Colon site by } \\
\text { gender }\end{array}$ & $\begin{array}{c}\text { KRAS- } \\
\text { mutated } \\
\text { CRC }\end{array}$ & $\begin{array}{c}\text { BRAF- } \\
\text { mutated } \\
\text { CRC }\end{array}$ & $\begin{array}{l}\text { Null } \\
\text { CRC }\end{array}$ & Total & $\mathrm{P}$-value \\
\hline \multicolumn{6}{|l|}{ Females } \\
\hline Cecum & $20(33)$ & $18(30)$ & $22(37)$ & 60 & \\
\hline $\begin{array}{l}\text { Ascending } \\
\text { colon }\end{array}$ & 10 (18) & $29(51)$ & $18(32)$ & 57 & \\
\hline $\begin{array}{l}\text { Transverse } \\
\text { colon }\end{array}$ & $13(41)$ & $9(28)$ & $10(31)$ & 32 & \\
\hline $\begin{array}{l}\text { Descending } \\
\text { colon }\end{array}$ & 6 (35) & $7(41)$ & $4(24)$ & 17 & \\
\hline $\begin{array}{l}\text { Sigmoid } \\
\text { colon }\end{array}$ & $15(20)$ & $5(7)$ & $56(74)$ & 76 & \\
\hline Rectum & $27(25)$ & $11(10)$ & 70 (65) & 108 & \\
\hline \multicolumn{6}{|l|}{ Males } \\
\hline Cecum & $23(46)$ & $7(14)$ & $20(40)$ & 50 & \\
\hline $\begin{array}{l}\text { Ascending } \\
\text { colon }\end{array}$ & $15(35)$ & $10(23)$ & $18(42)$ & 43 & \\
\hline $\begin{array}{l}\text { Transverse } \\
\text { colon }\end{array}$ & $5(16)$ & $4(13)$ & $22(71)$ & 31 & \\
\hline $\begin{array}{l}\text { Descending } \\
\text { colon }\end{array}$ & $1(10)$ & $1(10)$ & $8(80)$ & 10 & 0.02 \\
\hline $\begin{array}{l}\text { Sigmoid } \\
\text { colon }\end{array}$ & $29(32)$ & $8(9)$ & $55(60)$ & 92 & \\
\hline Rectum & 46 (29) & $11(7)$ & $103(64)$ & 160 & \\
\hline \multicolumn{6}{|l|}{ All patients } \\
\hline Cecum & $43(39)$ & $25(23)$ & $42(38)$ & 110 & \\
\hline $\begin{array}{l}\text { Ascending } \\
\text { colon }\end{array}$ & $25(25)$ & 39 (39) & 36 (36) & 100 & \\
\hline $\begin{array}{l}\text { Transverse } \\
\text { colon }\end{array}$ & $18(29)$ & $13(21)$ & $32(51)$ & 63 & \\
\hline $\begin{array}{l}\text { Descending } \\
\text { colon }\end{array}$ & $7(26)$ & $8(30)$ & $12(44)$ & 27 & \\
\hline $\begin{array}{l}\text { Sigmoid } \\
\text { colon }\end{array}$ & $44(26)$ & $13(8)$ & 111 (66) & 168 & \\
\hline Rectum & $73(27)$ & $22(8)$ & $173(65)$ & 268 & \\
\hline
\end{tabular}

${ }^{\text {a }} P$-value comparing male vs female subjects for nadir region $K R A S$ mutated carcinomas.

Results are given in absolute number and (percentage). The nadir region in the transverse colon and descending colon where absolute numbers are in bold.

tumors) (Table 1). BRAF-mutated carcinoma were more likely to be found in the proximal colon than were KRAS-mutated or -null carcinomas $(64 \%$ vs $41 \%$ and $27 \%$, respectively; $P<0.001$ for $K R A S-v S$ $B R A F$-mutated carcinoma). In addition, KRAS-mutated carcinomas were significantly more proximally located than were the null carcinomas (41 vs $27 \% ; P=0.001)$. Distributions with respect to primary sites in the large bowel for all three tumor subgroups (KRAS-mutated, BRAF-mutated and null carcinomas) are shown in Table 2 and Figure 1, overall and stratified for gender. The absolute number of tumors varied greatly along the proximal-distal axis of the large bowel, following a bimodal distribution with a nadir present in the transverse and descending colon segments. This pattern was also evident for KRAS-mutated
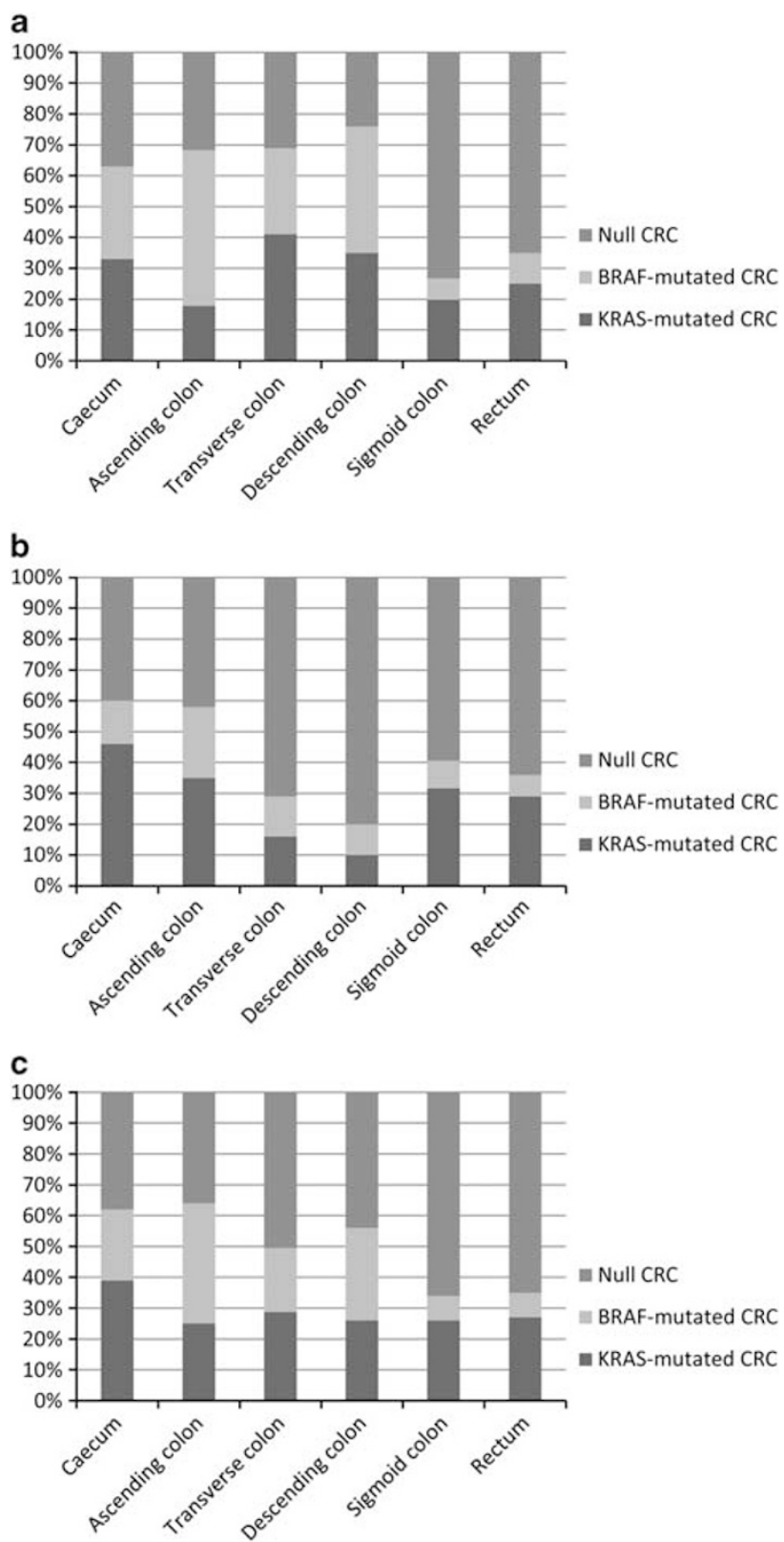

Figure 1 Relative proportions of colorectal carcinomas in different segments of the colorectum in relation to $B R A F$ mutation, KRAS mutation, non-KRAS or BRAF mutation (null carcinoma) in female patients (a), male patients (b) and in all patients (c).

carcinomas overall and in male subjects. In female subjects, although the absolute numbers of tumors also followed this bimodal pattern, there were differences in proportions when compared with male subjects. The proportion of KRAS-mutated carcinomas was greater in female subjects in the transverse colon and the descending colon compared with male subjects $(19 / 49$ or $39 \%$ KRASmutated carcinomas in female subjects vs 6/41 or $15 \%$ in male subjects; $P=0.02$ ). A significantly increased frequency of KRAS-mutated carcinoma was seen in the cecum (39 vs $26 \%$ in the remaining colorectum; $P=0.01)$. 


\section{Morphological characteristics}

Pathology features are summarized in detail in Table 1. Both KRAS- and BRAF-mutated carcinomas more frequently demonstrated focal or predominant mucinous differentiation when compared to null carcinomas $(46 \%$ and $52 \%$ respectively vs $25 \%$; $P<0.001$ for KRAS-mutated carcinomas vs null carcinomas). The presence of a contiguous residual polyp was more frequent in KRAS-mutated tumors than in other tumor subsets $38 \%$ in $K R A S$-mutated carcinomas vs $18 \%$ in $B R A F$-mutated carcinomas and $22 \%$ of null carcinomas; $P<0.001$ for both comparisons). However, the association between KRAS mutation and the presence of a residual contiguous polyp was only significant in male subjects. A contiguous polyp was present in $44 \%$ KRAS-mutated tumors compared with 18\% KRAS wild-type tumors in male subjects $(P<0.001)$, whereas it was found in 31\% KRAS-mutated tumors and $24 \%$ KRAS wild-type tumors in female subjects $(P=0.21)$. The distribution of residual polyp histological types was different among tumor subsets (Table 1). Contiguous polyps demonstrated a villous component in $99 \%$ of KRAS-mutated carcinomas compared with $81 \%$ of all other carcinomas $(P=0.001)$, and $78 \%$ of null carcinomas $(P=0.001)$. The histological subtype of contiguous polyp in KRAS-mutated tumors was tubulovillous adenoma in $79 \%$, villous adenoma in $13 \%$, traditional serrated adenoma in $8 \%$, and tubular adenoma in 1\%. BRAF-mutated tumors demonstrated a villous component in all but one of the observed contiguous polyps. No significant difference was found between codon 12 KRAS-mutated carcinoma and codon 13 $K R A S$-mutated carcinoma for any pathological features $(P>0.05$ in all instances; Supplementary Table).

\section{Molecular Pathology Findings}

Molecular pathway-associated findings are listed in Table 1. The partition of colorectal carcinoma into three MSI subclasses resulted in significant differences between KRAS-mutated carcinoma and KRAS wild-type carcinoma $(P<0.001)$, with $16 \%$ of $K R A S$ mutated tumors showing MSI-low/indeterminate phenotype compared with $10 \%$ in KRAS wild-type tumors. There was an increased frequency of $M G M T$ methylation in KRAS-mutated carcinomas compared with null carcinomas and $B R A F$-mutated carcinomas $(47 \%$ VS $26 \%$ and $12 \% ; P=0.01$ and 0.001, respectively). Loss of immunohistochemical expression of MGMT was present in $22 \%$ of all tumors and was also more common in KRAS-mutated carcinomas compared with KRAS wild-type carcinomas (27 vs 20\%; $P=0.05)$. Of colorectal carcinomas with normal MGMT methylation status, $81 \%$ demonstrated no loss of immunohistochemical expression of MGMT. This level contrasts significantly with MGMT

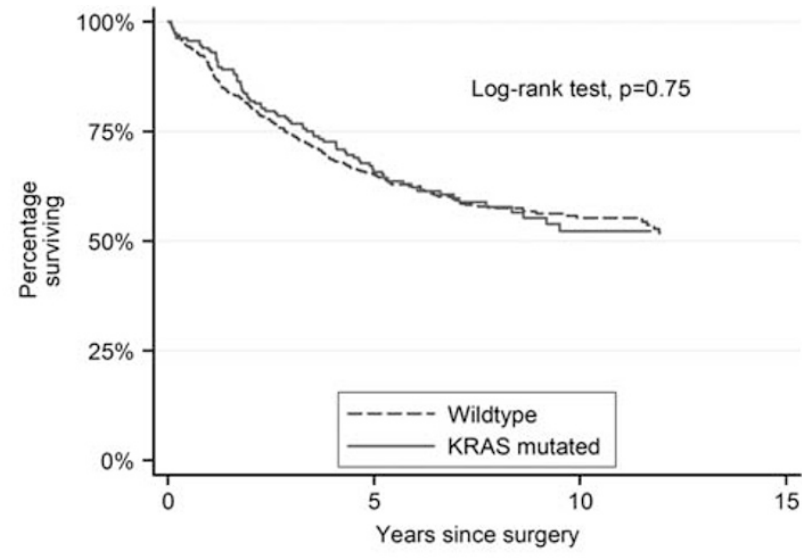

Number at risk

Wildtype 481 KRAS mutated 186 236 103 33

12

Figure 2 Kaplan-Meier curves comparing overall survival of 667 patients with colorectal carcinoma patients according to KRAS status (wild-type and mutated).

methylation-positive tumors where $43 \%$ showed no loss of expression $(P<0.001)$. No significant difference was found between codon $12 \mathrm{KRAS}$ mutated carcinoma and codon 13 KRAS-mutated carcinoma for any molecular features $(P>0.05$ in all instances; Supplementary Table).

\section{Statistical Adjustment for Multiple Testing}

The $P$-value for significance was adjusted for multiple hypothesis testing using the Bonferroni correction for comparisons of 17 variables (16 comparisons between KRAS mutation and other variables in Table 1 and one additional comparison in Table 2) to $P=0.05 / 34=0.0015$. The level of significance was considered as borderline if the $P$-value lay between 0.05 and 0.0015 after this correction was applied. The associations that remained significant after the correction was applied between KRASmutated tumors and one of more other tumor subtypes include mucinous differentiation, presence of a contiguous polyp, different MSI status and MGMT methylation (Table 1).

\section{Survival Analyses}

Among the 667 (86\%) of the 776 patients with data available on all relevant variables, there were 272 deaths during a median follow-up of 7.84 years (range 4.8 months-16.7 years) for censored patients. Figure 2 shows the estimated Kaplan-Meier survival curves by KRAS mutation status. There is no evidence of a difference in survival between these groups $(P=0.75)$. We found no evidence of a violation of the proportional hazards assumption. In our multivariable Cox regression model, $B R A F$ mutation was strongly associated with poorer survival, $\mathrm{HR}=1.46$ (95\% CI: 1.03, 2.06), $P=0.03$. 
Conversely, KRAS-mutated tumor patients demonstrated no statistically significant difference in overall survival, $\mathrm{HR}=0.99$ (95\% CI: 0.75, 1.32), $P=0.97$. Further grouping KRAS status by codon mutation subtype showed no evidence of an association between KRAS status and survival. Excluding patients with a $B R A F$ mutation did not affect these conclusions.

\section{Discussion}

In this report, we examined the pathological and molecular features of KRAS-mutated colorectal carcinoma in a population-based cohort of 776 patients. The observed features were compared with those of $B R A F$-mutated carcinomas and with tumors demonstrating neither $B R A F$ nor KRAS mutation (null carcinomas). Our exon 2 KRAS mutation rate of $28 \%$ is in agreement with previous studies when mutations in exon 2 only are considered.10,11,16,17 Like $B R A F$-mutated carcinomas, KRAS-mutated carcinomas showed an increased frequency of mucinous differentiation. This finding has been previously observed by Lin et $a l^{28}$ and more recently by Pai et $a l,{ }^{8}$ who reported that $32 \%$ of proximal KRAS-mutated carcinoma showed mucinous differentiation compared with $25 \%$ of null carcinomas. Maltzman et $a l^{29}$ previously reported that patients with KRAS mutated adenomas were significantly older than adenoma patients without a KRAS mutation. In our study, we did not find significant age difference between patients with a KRAS-mutated carcinoma and other carcinoma patients.

One of the novel findings of our study is the bimodal distribution of colorectal carcinoma frequency along the proximal-distal axis of the large bowel. Consistent with a recent report, we found the highest incidence of site-specific KRAS-mutated tumors in the cecum with the frequency of KRASmutated carcinoma significantly higher in the cecum $(39 \%)$ than in the rest of the colorectum (26\%), although not as high as the figure reported by Yamauchi et $\mathrm{al}^{30}(52 \%)$. We also found variations in proportions of KRAS-mutated carcinoma in different colonic segments between male and female subjects, which did not constitute a continuum. Although we cannot exclude that the observed results are chance findings due to low case numbers, this result raises interesting hypotheses. Epidemiological studies also reported different lifestyle risk factors in male and female subjects with a KRAS-mutated colorectal carcinoma. ${ }^{31}$ Possible explanations for both distribution variation along the colon and molecular subset variation between genders are likely to be multifactorial and complex. It may include the effect of lifestyle risk factors and host interaction with biochemical and bacterial luminal microenvironments.

It has long been observed that luminal environmental conditions alter from the distal ileum to the rectum, and that these conditions are associated with changes in the physical chemistry of the lumen contents including $\mathrm{pH}$, proportions of short- and branched-chain fatty acids and the products of protein fermentation, ${ }^{32}$ as well as those of the microbiota. Colorectal bacterial populations are responsible for important aspects of both health (synthesis of micronutrients such as Vitamin K, protection of the intestine from pathogenic species) and disease (inflammatory reactions and production of carcinogenic metabolites). In relatively recent publications, authors have described associations between species of bacteria, such as Streptococcus bovis subtypes, ${ }^{33}$ and Fusobacterium spp. ${ }^{34}$ and colorectal neoplasia. Importantly, the composition of the gut microbiota also varies with gender, ${ }^{34}$ and this may be reflected in the distinct distributions of molecular colorectal carcinoma subtypes seen in male and female subjects. A possible explanation for the bimodal distribution of colorectal carcinoma, we observed is that the contact of the large bowel mucosa with luminal contents may last longer in both extremities of the colon than in the mid-colon. Contractions originating in the transverse colon frequently move towards the cecum as well as distally, allowing the proximal colon to act as a braking mechanism on the forward movement of waste products entering from the small intestine, thus ensuring adequate absorption of salts and water. $^{35}$ This mechanism might produce areas of stasis at the extremities of the large bowel and may be related to the bimodal distribution of tumors seen in this cohort.

The frequent presence of a contiguous polyp adjacent to KRAS-mutated carcinoma has not been reported previously. Differences in tissue specimen handling from multiple sources may partially affect this result. The large number of cases analyzed and the robust statistical association after multiple comparison adjustment suggest a significant finding. However, the biological relevance of this association is unclear, in particular the difference observed between males and female patients. Separate studies need to be performed to verify our results. Possible explanations are that KRAS-mutated colorectal carcinoma may require contiguity with their precursor polyps to progress and/or that the malignant component may develop slowly and not rapidly overgrow its precursor lesion. Interestingly, increases in frequency of residual polyp adjacent to carcinoma have also been reported for biallelic and compound heterozygote MUTYH mutation carriers. ${ }^{36}$ An important feature of the tumors in those particular patients is the high rate of KRAS mutation, with over $60 \%$ of tumors harboring the c.35G $>$ A transition. ${ }^{37}$

Only a minority of adenomatous and serrated polyps have the capacity for malignant transformation. Adenomas with a villous component comprise a minority of colonic polyps but have an increased risk of malignant transformation. ${ }^{38}$ An association 
between KRAS mutation and the presence of a villous component in adenomas has previously been reported by multiple authors. ${ }^{19,29,39}$ Interestingly, the majority of contiguous polyps in $B R A F$-mutated carcinoma from our study were traditional serrated adenoma and tubulovillous adenoma, while none of these tumors demonstrated features of sessile serrated adenoma.

Initial attempts to understand the development of colorectal carcinoma were based on the traditional adenoma-carcinoma model, and were greatly expanded by the molecular analyses of Vogelstein and co-worker in $1988 .{ }^{19}$ This was augmented by the slow recognition of an alternative serrated neoplasia pathway over a decade later, initially from mucin expression studies. ${ }^{40}$ These canonical pathways are largely linear in nature, and are likely to serve as the foundation of colorectal carcinoma development in the majority of instances. In 2006, Jass et $a l^{41}$ proposed a 'fusion pathway', which combined elements from the canonical pathways. It was suggested that other mutational alterations, such as the inactivation of MGMT, may combine with KRAS and TP53 mutations to produce malignant transformation. KRAS mutation, as well as low/indeterminate levels of MSI and CIMP, has been associated with methylation of MGMT by several authors. ${ }^{21,22,42,43}$ In our study, we found that some KRAS-mutated carcinomas were associated with MGMT immunohistochemical loss of expression (borderline significance), MGMT methylation, and MSI-low/indeterminate phenotype. Of note, we found poor correlation between MGMT immunohistochemical loss of expression and MGMT methylation, as reported by others in colorectal carcinoma and glioblastoma. ${ }^{44,45}$ This can be secondary to various causes including methodology issues (type of methylation assay, intratumor heterogeneity) and alternative molecular mechanisms that regulate MGMT expression.

Overall, we did not demonstrate any significant effect of KRAS mutation subtypes (all mutations, codon 12 or codon 13) on patient survival, whether or not $B R A F$-mutated carcinomas were included in the analysis. This finding is different from Imamura et $a l,{ }^{17}$ who recently reported an association between poor survival and KRAS codon 12 mutation in BRAF wild-type colorectal carcinoma patients. With multiple studies showing different effects of KRAS mutation on patient survival, it is likely that various confounders may interact and that KRAS mutation does not represent on its own an important prognostic factor in patients with colorectal carcinoma.

In summary, we uncovered evidence that KRASmutated carcinomas demonstrate distinctive features from other colorectal carcinomas, in particular from tumors harboring neither BRAF nor KRAS mutation. To reduce the possibility of our novel associations being false-positive results, we reexamined the comparisons performed using a
Bonferroni correction procedure. Our key associations (mucinous differentiation, presence of a contiguous polyp, different MSI status and MGMT methylation) were robust to this correction for multiple hypothesis testing. Although some environmental and lifestyle risk factors, such as physical activity, body mass index, tobacco smoke and meat consumption, have been reported to be associated with KRAS-mutated colorectal carcinoma, ${ }^{31,46-49}$ potential new associations are unlikely to be identified if, as was the case for $B R A F$-mutated carcinomas until relatively recently, KRAS-mutated carcinomas continue to be included with the bulk of common colorectal carcinoma. Subsequent studies from different population are required to validate our findings.

\section{Acknowledgements}

We thank all study participants of the Melbourne Collaborative Cohort Study (NHMRC 509348) for their contributions to this project. We also acknowledge the contributions of Charmaine Smith, Lisa Oates and Sonia Terre'Blanche from the Cancer Council Victoria for their assistance with tissue block acquisition. This work was supported by the National Health and Medical Research Council and Cancer Council Victoria. During this work, JPY was a Cancer Council Queensland Senior Research Fellow. CR is a Jass Pathology Fellow. MAJ is a NHMRC Senior Research Fellow and JLH is a NHMRC Australia Fellow.

\section{Disclosure/conflict of interest}

The authors declare no conflict of interest.

\section{References}

1 Jass JR. Classification of colorectal cancer based on correlation of clinical, morphological and molecular features. Histopathology 2007;50:113-130.

2 Ogino S, Chan AT, Fuchs CS, et al. Molecular pathological epidemiology of colorectal neoplasia: an emerging transdisciplinary and interdisciplinary field. Gut 2011;60:397-411.

3 Jenkins MA, Hayashi S, O’Shea AM, et al. Pathology features in Bethesda guidelines predict colorectal cancer microsatellite instability: a population-based study. Gastroenterology 2007;133:48-56.

4 English DR, Young JP, Simpson JA, et al. Ethnicity and risk for colorectal cancers showing somatic BRAF V600E mutation or CpG island methylator phenotype. Cancer Epidemiol Biomarkers Prev 2008;17: 1774-1780.

5 Samowitz WS, Albertsen H, Sweeney C, et al. Association of smoking, $\mathrm{CpG}$ island methylator phenotype, and V600E BRAF mutations in colon cancer. J Natl Cancer Inst 2006;98:1731-1738. 
6 Ogino S, Fuchs CS, Giovannucci E. How many molecular subtypes? Implications of the unique tumor principle in personalized medicine. Expert Rev Mol Diagn 2012;12:621-628.

7 Cancer Genome Atlas Network. Comprehensive molecular characterization of human colon and rectal cancer. Nature 2012;487:330-337.

8 Pai RK, Jayachandran P, Koong AC, et al. BRAFmutated, microsatellite-stable adenocarcinoma of the proximal colon: an aggressive adenocarcinoma with poor survival, mucinous differentiation, and adverse morphologic features. Am J Surg Pathol 2012;36: 744-752.

9 Hughes LA, Williamson EJ, van Engeland M, et al. Body size and risk for colorectal cancers showing BRAF mutations or microsatellite instability: a pooled analysis. Int J Epidemiol 2012;41:1060-1072.

10 Nosho K, Irahara N, Shima K, et al. Comprehensive biostatistical analysis of $\mathrm{CpG}$ island methylator phenotype in colorectal cancer using a large populationbased sample. PLoS One 2008;3:e3698.

11 Samowitz WS, Curtin K, Schaffer D, et al. Relationship of Ki-ras mutations in colon cancers to tumor location, stage, and survival: a population-based study. Cancer Epidemiol Biomarkers Prev 2000;9:1193-1197.

12 Bos JL, Fearon ER, Hamilton SR, et al. Prevalence of ras gene mutations in human colorectal cancers. Nature 1987;327:293-297.

13 Roth AD, Tejpar S, Delorenzi M, et al. Prognostic role of KRAS and BRAF in stage II and III resected colon cancer: results of the translational study on the PETACC-3, EORTC 40993, SAKK 60-00 trial. J Clin Oncol 2010;28:466-474.

14 Westra JL, Schaapveld M, Hollema H, et al. Determination of TP53 mutation is more relevant than microsatellite instability status for the prediction of diseasefree survival in adjuvant-treated stage III colon cancer patients. J Clin Oncol 2005;23:5635-5643.

15 Richman SD, Seymour MT, Chambers P, et al. KRAS and BRAF mutations in advanced colorectal cancer are associated with poor prognosis but do not preclude benefit from oxaliplatin or irinotecan: results from the MRC FOCUS trial. J Clin Oncol 2009;27:5931-5937.

16 Andreyev HJ, Norman AR, Cunningham D, et al. Kirsten ras mutations in patients with colorectal cancer: the 'RASCAL II' study. Br J Cancer 2001; 85:692-696.

17 Imamura Y, Morikawa $\mathrm{T}$, Liao X, et al. Specific mutations in KRAS codons 12 and 13, and patient prognosis in 1075 BRAF wild-type colorectal cancers. Clin Cancer Res 2012;18:4753-4763.

18 Karapetis CS, Khambata-Ford S, Jonker DJ, et al. K-ras mutations and benefit from cetuximab in advanced colorectal cancer. N Engl J Med 2008;359:1757-1765.

19 Vogelstein B, Fearon ER, Hamilton SR, et al. Genetic alterations during colorectal-tumor development. N Engl J Med 1988;319:525-532.

20 Smith G, Carey FA, Beattie J, et al. Mutations in APC, Kirsten-ras, and p53-alternative genetic pathways to colorectal cancer. Proc Natl Acad Sci USA 2002;99:9433-9438.

21 Ogino S, Kawasaki T, Kirkner GJ, et al. CpG island methylator phenotype-low (CIMP-low) in colorectal cancer: possible associations with male sex and KRAS mutations. J Mol Diagn 2006;8:582-588.

22 Whitehall VL, Walsh MD, Young J, et al. Methylation of $O$-6-methylguanine DNA methyltransferase characterizes a subset of colorectal cancer with lowlevel DNA microsatellite instability. Cancer Res 2001; 61:827-830.

23 Giles GG, English DR. The Melbourne Collaborative Cohort Study. IARC Sci Publ 2002;156:69-70.

24 Hamilton SR, Bosman FT, Boffetta P, et al. Carcinoma of the colon and rectum, In: Bosman FT, Carneiro F, Hruban RH, Theise ND(eds) WHO Classification of Tumours of the Digestive System. IARC Press: Lyon, France; 2010, pp 134-146.

25 Rosty C, Buchanan DD, Walsh MD, et al. Phenotype and polyp landscape in serrated polyposis syndrome: a series of 100 patients from genetics clinics. Am J Surg Pathol 2012;36:876-882.

26 Newcomb PA, Baron J, Cotterchio M, et al. Colon Cancer Family Registry: an international resource for studies of the genetic epidemiology of colon cancer. Cancer Epidemiol Biomarkers Prev 2007;16: 2331-2343.

27 Weisenberger DJ, Siegmund KD, Campan M, et al. CpG island methylator phenotype underlies sporadic microsatellite instability and is tightly associated with BRAF mutation in colorectal cancer. Nat Genet 2006; 38:787-793.

28 Lin JK, Chang SC, Wang HS, et al. Distinctive clinicopathological features of Ki-ras mutated colorectal cancers. J Surg Oncol 2006;94:234-241.

29 Maltzman T, Knoll K, Martinez ME, et al. Ki-ras protooncogene mutations in sporadic colorectal adenomas: relationship to histologic and clinical characteristics. Gastroenterology 2001;121:302-309.

30 Yamauchi M, Morikawa T, Kuchiba A, et al. Assessment of colorectal cancer molecular features along bowel subsites challenges the conception of distinct dichotomy of proximal versus distal colorectum. Gut 2012;61:847-854.

31 Slattery ML, Anderson K, Curtin K, et al. Lifestyle factors and Ki-ras mutations in colon cancer tumors. Mutat Res 2001;483:73-81.

32 Macfarlane GT, Gibson GR, Cummings JH. Comparison of fermentation reactions in different regions of the human colon. J Appl Bacteriol 1992;72:57-64.

33 Abdulamir AS, Hafidh RR, Abu Bakar F. The association of Streptococcus bovis/gallolyticus with colorectal tumors: the nature and the underlying mechanisms of its etiological role. J Exp Clin Cancer Res 2011;30:11.

34 Kostic AD, Gevers D, Pedamallu CS, et al. Genomic analysis identifies association of Fusobacterium with colorectal carcinoma. Genome Res 2012;22: 292-298.

35 Reddy SN, Bazzocchi G, Chan S, et al. Colonic motility and transit in health and ulcerative colitis. Gastroenterology 1991;101:1289-1297.

36 Cleary SP, Cotterchio M, Jenkins MA, et al. Germline MutY human homologue mutations and colorectal cancer: a multisite case-control study. Gastroenterology 2009;136:1251-1260.

37 Lipton L, Halford SE, Johnson V, et al. Carcinogenesis in MYH-associated polyposis follows a distinct genetic pathway. Cancer Res 2003;63:7595-7599.

38 Martinez ME, Baron JA, Lieberman DA, et al. A pooled analysis of advanced colorectal neoplasia diagnoses after colonoscopic polypectomy. Gastroenterology 2009;136:832-841.

39 Yuen ST, Davies H, Chan TL, et al. Similarity of the phenotypic patterns associated with BRAF and KRAS 
mutations in colorectal neoplasia. Cancer Res 2002;62:6451-6455.

40 Biemer-Huttmann AE, Walsh MD, McGuckin MA, et al. Mucin core protein expression in colorectal cancers with high levels of microsatellite instability indicates a novel pathway of morphogenesis. Clin Cancer Res 2000;6:1909-1916.

41 Jass JR, Baker K, Zlobec I, et al. Advanced colorectal polyps with the molecular and morphological features of serrated polyps and adenomas: concept of a 'fusion' pathway to colorectal cancer. Histopathology 2006; 49:121-131.

42 Nagasaka T, Sasamoto H, Notohara K, et al. Colorectal cancer with mutation in BRAF, KRAS, and wild-type with respect to both oncogenes showing different patterns of DNA methylation. J Clin Oncol 2004;22: 4584-4594.

43 Ogino S, Kawasaki T, Kirkner GJ, et al. Molecular correlates with MGMT promoter methylation and silencing support $\mathrm{CpG}$ island methylator phenotypelow (CIMP-low) in colorectal cancer. Gut 2007;56: 1564-1571.
44 Rodriguez FJ, Thibodeau SN, Jenkins RB, et al. MGMT immunohistochemical expression and promoter methylation in human glioblastoma. Appl Immunohistochem Mol Morphol 2008;16:59-65.

45 Shima K, Morikawa T, Baba Y, et al. MGMT promoter methylation, loss of expression and prognosis in 855 colorectal cancers. Cancer Causes Control 2011;22:301-309.

46 Curtin K, Samowitz WS, Wolff RK, et al. Somatic alterations, metabolizing genes and smoking in rectal cancer. Int J Cancer 2009;125:158-164.

47 Brink M, Weijenberg MP, de Goeij AF, et al. Meat consumption and K-ras mutations in sporadic colon and rectal cancer in The Netherlands Cohort Study. Br J Cancer 2005;92:1310-1320.

48 Samadder NJ, Vierkant RA, Tillmans LS, et al. Cigarette smoking and colorectal cancer risk by KRAS mutation status among older women. Am J Gastroenterol 2012;107:782-789.

49 Lee JE, Baba Y, Ng K, et al. Statin use and colorectal cancer risk according to molecular subtypes in two large prospective cohort studies. Cancer Prev Res (Phila) 2011;4:1808-1815.

Supplementary Information accompanies the paper on Modern Pathology website (http://www.nature.com/ modpathol) 